\title{
Comparison of clinical applications of single-dose intravenous injection of mivacurium and cisatracurium in adults' vocalcordpolyps resection under self-retaining laryngoscope
}

\author{
Fanglei Han, Lu Zhang, Shuang Cheng and Feng Liang ${ }^{a}$ \\ China-Japan Union Hospital of Jilin University
}

\begin{abstract}
Objective: Through comparing the clinical observation of mivacurium and cisatracurium in vocal polyp extraction, to study the advantage of mivacurium in vocal polyp extraction. Methods:Forty American Society of Anesthesiologists(ASA) physical status I II patients for vocal polyp extraction,aged 18 60 years old, were randomly divided into two groups as Mivacurium injection group(Group M) and Cis-atracurium injection group(Group C), each group includes 20 subjects. None of the patients are allergic, has serious diseases of cardiovascular system,liver or kidney.None of them has asthma, airway high response, difficult airway or neuromuscular diseases.Those patients who use beta-blockers or calcium channel blockers for a long time were excluded .All the subjects had the same premedication, fasting and fluid fobidden time.All the subjects who get into the operating room get the routine monitoring of electrocardiogram(EEG), blood pressure(BP), heart rate(HR), pulse oxygen saturation(SpO2) and the TOF WATCH SX. Each group gives the same medicine other than the muscle relaxant during induction of general anesthesia to do the vocal polyp extraction by the same experienced operator. Two groups were recorded in each index of anesthesia induction and tracheal intubation conditions, operation conditions, anesthesia, muscle relaxation monitoring.Results: There are no statistically significant in Cormack-Lehane grading system,Cooper's grading system and operation satifaction( $p>0.05)$. 2.Group A have shorter intubation time than Group $\mathrm{B}(\mathrm{p}<0.05)$.3.In the TOF recovery time to $25 \%$ and $90 \%$, extubation time, the $\mathrm{M}$ group was faster than the $\mathrm{C}$ group, and the difference was statistically significant $(\mathrm{p}<0.05)$.Conclusion: 1.A single intubating dose of mivacurium can provide similar intubation and surgeon satisfaction for the vocal polyp extraction.2.Compared with cis-atracurium, mivacurium can shorten the intubation time and the recovery time of anesthesia. The adverse reactions of mivacurium is mild, and it has less Residual muscle relaxation. Therefore mivacurium is more suitable for the vocal polyp extraction than cis-atracurium.
\end{abstract}

\section{Instruction}

Vocalcordpolyps is a kind of benign neoplasm commonly seen in pharynx and larynx and its typical clinical manifestation is voice hoarseness of various degrees. There is an upward trend in its incidence in recent years ${ }^{[1]}$. Clinically, it is mainly treated by operations [2]. The vocalcordpolyps extracation under self-retaining laryngoscope has been widely applied in clinical practice because of its unique advantages. This surgical operation only takes a short time and surgical spots are in pharynx and larynx, which will be strongly pungent. Thus, it requires enough depth of anesthesia. During the

\footnotetext{
${ }^{a}$ Corresponding author: 295253720@qq.com
} 
operation, patients shall have stable life signs and they shall be able to wake up quickly after the operation. Patients are required to be wake up smoothly and steadily. Thus, this surgical method has a high requirement to anesthesia degree and it is also extremely important to select a safe and short-acting muscle relaxing drug. This paper conducted a comparative clinical observation to the single-dose intravenous injection effect of two muscle relaxing drugs (cisatracurium and mivacurium) when they were used to vocalcordpolyps extracation under self-retaining laryngoscope so as to find a safer, more effective muscle relaxing drug with high satisfaction degrees in the surgical operation.

\section{Materials and Methods}

Patients (male or female) aged 18 60 years old who would undergo vocalcordpolyps extracation under self-retaining laryngoscope some time in our hospital were selected. They belonged to level I II of American Society of Anesthesiologists (ASA). 40 cases were selected, including 20 male cases and 20 female cases. The patients were randomly divided into two groups (with 20 patients in each group): mivacurium induced group (Group $\mathrm{M}$ ) and cisatracurium group (Group C).

Methods:All patients were forbidden to eat anything within 8 hours and drink anything within four hours before the operation. Before the operation, they all took penehyclidine hydrochloride of $1.0 \mathrm{mg}$. After the patients came to the operating room, their venous channels of upper limb would be opened and the patient monitor would be connected. During the operation, their ECG, BP, HR and SpO2 were routinely monitored. Then, the TOF-Watch would be set and connected and the TOF stimulus mode would be used. The impulse current was $60 \mathrm{~mA}$, the frequency was $2 \mathrm{~Hz}$ and the stimulus interval was $15 \mathrm{~s}$. After the patients had stable life signs, the anesthesia induction would be conducted. Midazolam of $0.04 \mathrm{mg} / \mathrm{kg}$, propofol of $2 \mathrm{mg} / \mathrm{kg}$, fentanyl of $4 \mathrm{ug} / \mathrm{kg}$ and dexamethasone of $10 \mathrm{mg}$ were injected to their veins. After eyelash reflexes of patients disappeared, muscle relaxant calibration shall be done. The mode of train-of-four stimulation shall be used and the percentage of train-of-four stimulation shall be adjusted to $100 \%$ or so, which was also the baseline value. Then, mivacurium of $0.2 \mathrm{mg} / \mathrm{kg}$ would be injected with the injection speed of $45 \mathrm{~s}$ to patients of Group $\mathrm{M}$ while cisatracurium of $0.1 \mathrm{mg} / \mathrm{kg}$ would be injected to patients of Group C. When the TOF reaction number reduced to 2 or below, oral intubation under direct vision could be done. During the operation, sevoflurane was used for anesthesia and the MAC value was maintained to be between 1.2 and 1.3. When the mouth gags were taken out, operations of the two groups would be ended and at that time, the sevoflurane shall be stopped. As long as TOP of Group C went back to more than $25 \%$, neostigmine of $1 \mathrm{mg}$ and atropine of $0.5 \mathrm{mg}$ could be used for antagonism. Antagonism was not done for Group M. After the patients recovered their spontaneous respiration and swallowing reflex, their deoxidation $\mathrm{SpO} 2$ shall be maintained to above $90 \%$ for three minutes. The endotracheal tubes could be pulled out as long as they recovered consciousness. After that, they would be moved to the post-operational care unit (PACU) for continual observation. If the patients recovered their consciousness, could complete simple actions according to instructions (such as raising their head and sticking out their tongue), returned their cough reflex to normal level, had stable life signs and Steward grade of higher than 4 points and $\mathrm{TOF}>90 \%$ and showed no attenuation in terms of the train of four stimulation, they could be sent back to the department for monitoring care and oxygen inhalation.Observe and record the intubation time, the operation time, the extubation time and the PACU time, the time used for TOF value recovering to $25 \%$ (T25), the time used for TOF value recovering from $25 \%$ to $75 \%$ (recovery index) and the time used for TOF value recovering to $90 \%$ (T90).

SPSS 13.0 software was used for statistical analysis. All measurement data were shown by Mean \pm standard deviation and the variance analysis and T-test method were used for inter-group comparison. $\mathrm{P}<0.05$ meant that the difference showed statistical significance. Besides, chi-square test was used for enumeration data. $\mathrm{P}<0.05$ meant that the difference showed statistical significance. 


\section{Results}

The difference between Group $\mathrm{M}$ and Group $\mathrm{C}$ in their tracheal intubating conditions and operation conditions had no statistical significance $(\mathrm{p}>0.05)$ (refer to Table 1$)$

Table 1. Comparison of tracheal intubating conditions and operation conditions

\begin{tabular}{ccccc}
\hline & C-L grading & Irritating cough & Cooper's grading & $\begin{array}{c}\text { Operation condition } \\
\text { grading }\end{array}$ \\
\hline GROUP M & $19 / 1 / 0 / 0$ & $2 / 18$ & $17 / 3 / 0 / 0$ & $2 / 18$ \\
GROUP C & $18 / 2 / 0 / 0$ & $1 / 19$ & $16 / 3 / 1 / 1$ & $1 / 19$ \\
p value & $>0.05$ & $>0.05$ & $>0.05$ & $>0.05$ \\
\hline
\end{tabular}

Notes: The difference of inter-group comparison had no statistical significance. $\mathrm{p}>0.05$

Group M spend less time than Group C in terms of trachea anesthesia and intubation time, TOF 25\% and $90 \%$ recovery time, extubation time and PACU time. Besides, the difference showed statistical significance. (Refer to Table 2 and Table 3)

Table 2. Comparison of intubation time, operation time and extubation time

\begin{tabular}{clll}
\hline & Intubation time $(\mathrm{min})$ & Operation time $(\mathrm{min})$ & Extubation time $(\mathrm{min})$ \\
\hline GROUP M & $3.09 \pm 0.27$ & $12.69 \pm 1.74$ & $14.19 \pm 1.18$ \\
GROUP C & $3.62 \pm 0.44$ & $13.28 \pm 1.74$ & $23.09 \pm 4.79$ \\
p value & $<0.05$ & $>0.05$ & $<0.05$ \\
\hline
\end{tabular}

Notes: The difference of inter-group comparison had no statistical significance. $\mathrm{p}>0.05$

The difference of inter-group comparison had statistical significance. $p<0.05$

Table 3. Comparison of TOF value

\begin{tabular}{cccc}
\hline & T25 $(\min )$ & T90 $(\min )$ & Recovery index $(\min )$ \\
\hline GROUP M & $15.61 \pm 1.50$ & $22.09 \pm 2.68$ & $10.60 \pm 1.12$ \\
GROUP C & $32.57 \pm 1.44$ & $34.78 \pm 2.89$ & $4.55 \pm 1.09$ \\
p value & $<0.05$ & $<0.05$ & $<0.05$ \\
\hline
\end{tabular}

Notes: The difference of inter-group comparison had statistical significance. $\mathrm{P}<0.05$

\section{Discussion}

Using your voice too much or using it improperly, upper respiratory tract infection and contacting stimulating substances can all cause vocalcordpolyps and hoarseness of various degrees. Vocalcordpolyps is a benignant hyperplasic disease of vocal cords commonly seen in the E.N.T. department. At present, its main treatment method is surgical resection. Though this operation is short in time, it is strongly irritative and requires enough anesthesia depth. During the operation, patients shall have stable life signs and shall recover themselves rapidly and smoothly after the operation. Thus, it is especially important to select and use muscle-relaxing drugs because they shall work quickly and completely relax muscles within a short acting time. Besides, the patients shall recover quickly and have a small residual amount of muscle relaxant. This research studied the two types of muscle relaxant, hoping to compare them and find one which is more suitable for this surgery way.

Cisatracurium has strong muscle-relaxing effect and weak histamine release ${ }^{[3]}$ and it is widely used in clinic practice. Studies of HUANG Jiayang, etc ${ }^{[4]}$ showed that the application of co-induction of 2ED95 cisatracurium and sevoflurane to vocalcordpolyps extraction could give satisfied tracheal intubation conditions and help gain satisfactory muscle relaxant effect. Some studies ${ }^{[5]}$ showed that when the administration dosage is $0.1 \mathrm{mg} / \mathrm{g}(2 \mathrm{ED} 95)$, the onset time was about 3.0 minutes while other 
studies ${ }^{[6]}$ indicated that under the total intravenous anesthesia, if patients aged $18 \sim 75$ years old were injected with twice as much ED95 cisatracurium to their veins, the duration of their muscle relaxation was (32 \pm 7$)$ minutes on average. That was basically consistent with findings of this research. Based on the above results and comprehensively considering intubation conditions and maintenance time, twice as much ED95 cisatracurium was used for induction and tracheal intubation in this research.

Mivacurium is a kind of nondepolarizing muscle relaxant of Bis Benzylisoquinolines and is one of the most ideal short-acting muscle relaxants. It does not accumulate in the body and its action time is short. However, it can generate high plasma clearance rate without antagonism and it has no neurovirulence. Guo Chen [7] reported that mivacurium of $0.2 \mathrm{mg} / \mathrm{kg}$ could provide satisfactory intubation conditions and if the dosage increased to $0.25 \mathrm{mg} / \mathrm{kg}$, the working time could be obviously shortened. However, when the dose was more than $0.2 \mathrm{mg} / \mathrm{kg}$, the occurrence rate of erubescence would be raised significantly with the dosage increased. Thus, the inductive dose of mivacurium in this study was $0.2 \mathrm{mg} / \mathrm{kg}$ [8]. In anesthesia and inducing period of this research, the infusion speed was strictly controlled so as to reduce the histamine release and to ensure that it can be transfused for $45 \mathrm{~s}$ or longer. In addition, cisatracurium shall also be transfused to patients in the contrast group for $45 \mathrm{~s}$ or longer so as to ensure that muscle relaxant infusion speed of two groups was consistent.

Experiment results show that the difference of the two groups in terms of C-L grading, irritating cough, Cooper's grading and operation condition had no statistical significance, showing that muscle relaxants used in the experimental group and the contrast group and their dosage can meet the intubation condition and operation condition for vocalcordpolyps extracation under self-retaining laryngoscope. The intubation time, extubation time, the time need for TOF value recovering to $25 \%$ (T25), the time needed for TOF value recovering to $90 \%$ time (T90), the recovery index and the PACU time of Group $\mathrm{M}$ are all smaller than those of Group $\mathrm{C}$ and their difference has statistical significance $(\mathrm{p}<0.05)$, indicating that mivacurium of Group $\mathrm{M}$ works faster, needs shorter intubation time and its muscle relaxant recovery time is also shorter than that of Group C. Besides, its postoperative extubation time is also short, which can reduce patients' waiting time in the operating room and can be beneficial to shorten the operation cycle. The comparison difference of Steward analepsia score of patients of both groups has no statistical significance and this can ensure the anesthesia security of patients. In terms of adverse reactions of patients after their anesthesia induction and before their coming out of PACU, more patients in Group M are red in their faces and bodies than those in Group $\mathrm{C}$ and most flushing parts of them are in the chest. Patients in Group $\mathrm{C}$ have a higher occurrence rate of postoperative residual curarization, which means that, their TOF value is $<90 \%$ after tubes are pulled out. This is significantly higher than that in Group M, indicating that lower risks of postoperative pulmonary complications will exist and patients are safer if mivacurium is used. In this research, when muscle relaxants are used, patients need longer recovery time than that stated in the foregoing data. This is caused by the application of sevoflurane in the anesthesia maintenance stage because the sevoflurane has a synergetic muscle relaxant effect on muscle relaxants, which can affect the measurement of values of muscle relaxant itself. However, patients of both groups all use sevoflurane for anesthetic maintenance, the synergetic muscle relaxant effect caused by it can be ignored.

During the induction process, different muscle relaxant types are used to those two groups and other drugs are all used according to their weight in fixed quantity because this can avoid the influence of other drugs on the results. During the induction process, dexamethasone of $10 \mathrm{mg}$ is given routinely because it can reduce the histamine release of muscle relaxants of Bis Benzylisoquinolines on the one hand and can prevent the occurrence of postoperative edema of the larynx after the operation on the other hand.

In order to ensure the safety of patients, the following requirements shall be met when patients come out of the PACU: they shall be conscious and be able to complete simple actions according to instructions (such as raising their head and sticking out their tongue); they shall have stable life signs and their Steward score shall be larger than 4 points. Their TOF shall be larger than $90 \%$ and their train-of-four stimulation shall have no attenuation.

Vocal cord impairment extracation under self-retaining laryngoscope rather than the 
phonomicrosurgery technique is used as the surgical method in this research. This is a deficiency of this research in terms of its advancement. More surgical methods need to be done for researches of microsurgery anesthesia under self-retaining laryngoscope. This search also has the following shortcomings: patients of the two groups are between 18 and 60 years old and no research is done to children, the elderly, fat people or patients with internal medicine diseases.so, it has some limitations.

In conclusion, compared with cisatracurium, the application of mivacurium induction can shorten the anesthesia induction time and patients can recover quickly after the operation. Besides, they have milder adverse reactions and less muscle relaxant residuals. Thus, mivacurium induction is more applicable to vocalcordpolyps extracation under self-retaining laryngoscope.

\section{References}

1. Yingjun Shan, Jiawen Cui, Gang Fei, etal. Different surgical methods for the treatment of vocal cord polyp compared [J]. Tongji University, [Medical Edition]. 6(30):118-120(2009) (In Chinese)

2. Yingpeng Han, Chengjun Song, Xiuhui Jian, etal. Laryngeal endoscopic vocal cord polyp excision [J]. Chinese Journal of Otorhinolaryngology, 2(38):65(2009) (In Chinese)

3. Liqiu Mo, Wenqi Huang, Jiefang Tan, etal. A new effective muscle relaxant - cis - atracurium [J]. The Journal of Clinical Anesthesiology,16:170(2000) (In Chinese)

4. JiaYang Huang, Huizhang Liu, Guosheng Yan, etal. Different doses of cisatracurium and sevoflurane induced polypectomy in the application of vocal cord results [J], Practical Journal of Clinical Medicine, 189(23):187-188(2014) (In Chinese).

5. Ruijuan Wang. Comparison of the efficacy of cisapride atracurium in weightlessness and actual body weight [D], Southern Medical University, (2013) (In Chinese)

6. Yali Ma, Qingduo Guo, Rui Li, etal. Intravenous injection of cisatracurium on histamine release in patients with general anesthesia [J]. Zhonghua Anesthesiology, 29,(1):89-90(2009) (In Chinese)

7. Chen Guo, Mingjun Xu. Different doses of miracurium for gynecological laparoscopic muscle relaxation in clinical observation [J], Beijing Medical, 36(11):936-939(2014) (In Chinese)Yohitake Y,Shinohara M,Ue H,etal.Characteristics of Surface mec-hano myogran ale dependant on development of fusion of motor- units in humans[J].ApplPhysiol, 93(5):1744-1652 (2002). 